\title{
LUMINOSITY DISTRIBUTION IN cD CLUSTERS
}

\author{
F.W. BAIER \\ WIP - Working Group 'Galaxy Clusters' \\ University of Potsdam \\ An der Sternwarte 16 \\ 14482 Potsdam \\ Germany
}

The ratio of contributions from cannibalism and from cooling flows to the final $\mathrm{cD}$ galaxies in diverse clusters seems to be different. It should be determined by some general cluster properties as for instance the central mass concentration. Assuming that mass distribution is tantamount to luminosity distribution we have analyzed the question of possible luminosity segregation in the radial galaxy distribution of galaxy clusters (Baier \& Schmidt 1992; Baier \& MacGillivray 1994).

Three different types of $\mathrm{CD}$ clusters were found: clusters with radial luminosity segregation (segregation class S2); clusters without radial luminosity segregation (segregation class S1); and such objects exhibiting a phenomenon which we call antisegregation in luminosity, i.e. a deficiency of bright galaxies in the central regions of clusters (segregation class S0). The existence of these three classes is interpreted as evident proof of strong mass segregation (class S2), of moderate mass segregation (class S1), and of weak or absent mass segregation (class S0), respectively. These different segregation conditions in galaxy clusters appear to be indications for different evolutionary stages of these clusters. This result is in agreement with former investigations by Lugger $(1984,1989)$ who confirmed an inner/outer difference for the luminosity function of clusters of galaxies and especially a depletion of bright galaxies in the cores of some $\mathrm{cD}$ clusters, which might be the expected result of $\mathrm{CD}$ production by galactic cannibalism and accretion of material stripped from surrounding galaxies.

We await that the totality of all dominant galaxies observed at the present time should be a mixture of objects which formed at different epochs. Insofar the existence of dominant galaxies with quite different properties as well as correlations between these properties and cluster properties should be expected. Indeed there are correlations between the degree of central mass segregation and the properties of dominant galaxies. $\mathrm{CD}$ clusters with large peculiar $\mathrm{CD}$ velocities very often exhibit low or absent central mass segregation. On the other side, moderate and stronger mass segregation is observed mainly for clusters with small $\mathrm{CD}$ peculiar velocities. A substantial percentage of $\mathrm{CD}$ galaxies with large peculiar velocities has relative blue central regions. On the other hand there is a tendency for $\mathrm{CD}$ galaxies in clusters with strong mass segregation and low peculiar velocities to have slightly red centres or 'neutral' radial colour distributions without any pronounced gradient and low envelope luminosities similar to normal ellipticals. We consider such objects as in a very early evolutionary stage before any cannibalism and accretion of stripped material. The clusters with high luminosity $\mathrm{CD}$ envelope often belong to classes 1 and 0 . Accumulation of material stripped from cluster galaxies in galaxy-galaxy 
interactions should lead to the formation of $\mathrm{cD}$ envelopes. The envelope formation then should be followed by merging of the stripped galaxies with the dominant galaxy. The stripping process and the corresponding formation of the $\mathrm{CD}$ envelope in clusters of classes 1 and 0 should be advanced or completed, respectively.

A central dominant galaxy grows in luminosity with each merger but its velocity dispersion remains unchanged (Malamuth 1992). Therefore, a luminosity excess compared to the known $L \sim \sigma^{A}$ relation is expected for evolved $\mathrm{CD}$ galaxies. The amount of the residuals $\Delta L$ is considered as a measure of the dynamical evolution. It is interesting that A 401 and A 779 in segregation class 2 show none or small luminosity residuals only. On the other side A 2029 and A 2670 from segregation 1 and 0 show the highest luminosity residuals.

Porter et al. $(1988,1991)$ found shape breaks with respect to ellipticity and position angle profiles in most $\mathrm{CD}$ galaxy halos. The outer regions of BCGs are prolate and strongly aligned with the prolate cluster in which they lie. The authors see their findings to be in agreement with the scenario that BCGs are originally similar to normal ellipticals and then grow by accreting matter along the cluster axis.

Gudehus (1991) derived a connection $L \sim r^{0.95}$ between luminosity and radius of brightest cluster galaxies and compared it with the relation $L \sim r$ which is predicted by the theory of homologous merging. He grouped the dominant galaxies into equally spaced magnitude ranges of width 0.82 mag corresponding to galaxies of types $\mathrm{gE}, \mathrm{D}$ and $\mathrm{cD}$. The existence of different luminosity groups for dominant galaxies of types $\mathrm{gE}, \mathrm{D}$ and $\mathrm{CD}$ implicates a dynamical evolution from $\mathrm{gE}$ to $\mathrm{CD}$. Bhavsar (1989) concluded that the magnitudes of first ranked galaxies in rich clusters are best explained if they consist of two distinct populations: a population of special (i.e. evolved) galaxies and a population of extremes of a statistical luminosity function.

Evidence for significant velocity offsets of $\mathrm{cD}$ galaxies in clusters favours the idea that - at least many - cDs formed in poor and low-dispersion subclusters - which afterwards merged to produce rich clusters. In such a case the $\mathrm{CD}$ is not necessarily at rest at the centre of the merged cluster directly after the merging - although it was at the kinematic centre of the subcluster. This possibility is in agreement with the above discussed correlation between the peculiar velocity of the $\mathrm{CD}$ galaxy and the segregation class. It is plausible that $\mathrm{CDs}$ in subclusters before cluster merging should be in early evolutionary stages very often. On the other hand, cDs in merging and merged clusters (with higher peculiar velocities) should be more evolved by cannibalism and the following accretion of stripped material simple because of the advanced dynamical evolution.

The same focusing potential that can give rise to a cooling flow might attract the massive galaxy to the kinematical centre of the merged cluster, e.g. to the bottom of a deep cluster potential. At this unique position processes such as accretion of tidally-stripped material, cooling flows (and/or cannibalism) can be invoked in the final evolution state of the $\mathrm{cD}$ galaxy and should mainly influence the $\mathrm{CD}$ envelope. Therefore, it seems to be plausible that the envelope luminosity of a cD galaxy may provide an indication of the dynamical state of its host cluster (Bothun \& Schombert 1990). From the beginning of the subcluster merging until the final stage we should expect many intermediate evolutionary stages. The existence of such intermediate dynamical phases seems to be reflected by many observational results as the evidence for a high fraction of galaxy clusters with substructure, pronounced substructure even in cD clusters, bimodal and irregular $x$-ray surface brightness distributions, spatial offsets between the $c D s$ and the peaks of the $x$-ray emission, offsets between the $x$-ray peak position and the $x$-ray centre determined from the outermost contours of the $x$-ray images, twisting isophotes of the $x$-ray 
distribution in clusters, velocity offsets of the cDs, the existence of dumb-bell galaxies in cluster centres, etc.

\section{References}

Baier, F.W. and Schmidt, K.-H., 1992. Astron. Nachr., 313, 275.

Baier, F.W. and MacGillivray, H.T., 1994. Mon. Not. R. astron. Soc., in preparation.

Bhavsar, S.P., 1989. Astrophys. J., 338, 718.

Bothun, G.D. and Schombert, J.M., 1990. Astrophys. J., 360, 436.

Gudehus, D.H., 1991. Astrophys. J., 382, 1.

Lugger, P.M., 1984. Astrophys. J., 278, 51.

Lugger, P.M., 1989. Astrophys. J., 343, 572.

Malumuth, E.M., 1992. Astrophys. J., 386, 420.

Porter, A.C., 1988. PhD Thesis, Caltech.

Porter, A.C., Schneider, D.P. and Hoessel, J.G., 1991. Astron. J., 101, 1561. 